\title{
BMJ Open When is a randomised controlled trial health equity relevant? Development and validation of a conceptual framework
}

\author{
J Jull, ${ }^{1}$ M Whitehead, ${ }^{2}$ M Petticrew, ${ }^{3}$ E Kristjansson, ${ }^{4}$ D Gough, ${ }^{5}$ J Petkovic, ${ }^{6}$ \\ J Volmink, ${ }^{7,8}$ C Weijer, ${ }^{9}$ M Taljaard, ${ }^{10,11}$ S Edwards, ${ }^{12}$ L Mbuagbaw, ${ }^{13,14}$ \\ R Cookson, ${ }^{15} \mathrm{~J}$ McGowan, ${ }^{16}$ A Lyddiatt, ${ }^{17}$ Y Boyer, ${ }^{18}$ L G Cuervo, ${ }^{19}$ R Armstrong, ${ }^{20}$ \\ H White, ${ }^{21}$ M Yoganathan, ${ }^{6}$ T Pantoja, ${ }^{22}$ B Shea, ${ }^{6}$ K Pottie, O Norheim, ${ }^{23,24}$ \\ $\mathrm{S}$ Baird, ${ }^{25}$ B Robberstad, ${ }^{23,26} \mathrm{H}$ Sommerfelt, ${ }^{23,26,33} \mathrm{Y}^{\text {Asada, }}{ }^{27} \mathrm{G}$ Wells, ${ }^{28,29}$ \\ P Tugwell, ${ }^{6}$ V Welch ${ }^{6}$
}

To cite: Jull J, Whitehead M, Petticrew M, et al. When is a randomised controlled trial health equity relevant? Development and validation of a conceptual framework. BMJ Open 2017;7:e015815. doi:10.1136/ bmjopen-2016-015815

- Prepublication history and additional material for this paper are available online. To view please visit the journal (http:// dx.doi.org/10.1136/bmjopen2016-015815).

Received 3 January 2017 Revised 7 July 2017 Accepted 31 July 2017

\section{CrossMark}

For numbered affiliations see end of article.

Correspondence to

J Jull; jjull013@uottawa.ca

\section{ABSTRACT}

Background Randomised controlled trials can provide evidence relevant to assessing the equity impact of an intervention, but such information is often poorly reported. We describe a conceptual framework to identify health equity-relevant randomised trials with the aim of improving the design and reporting of such trials.

Methods An interdisciplinary and international research team engaged in an iterative consensus building process to develop and refine the conceptual framework via face-to-face meetings, teleconferences and email correspondence, including findings from a validation exercise whereby two independent reviewers used the emerging framework to classify a sample of randomised trials.

Results A randomised trial can usefully be classified as 'health equity relevant' if it assesses the effects of an intervention on the health or its determinants of either individuals or a population who experience ill health due to disadvantage defined across one or more social determinants of health. Health equity-relevant randomised trials can either exclusively focus on a single population or collect data potentially useful for assessing differential effects of the intervention across multiple populations experiencing different levels or types of social disadvantage. Trials that are not classified as 'health equity relevant' may nevertheless provide information that is indirectly relevant to assessing equity impact, including information about individual level variation unrelated to social disadvantage and potentially useful in secondary modelling studies.

Conclusion The conceptual framework may be used to design and report randomised trials. The framework could also be used for other study designs to contribute to the evidence base for improved health equity.

\section{INTRODUCTION}

Many countries have policy documents that reflect concern for social justice, with a goal to redressing disparities in health both within

\section{Strengths and limitations of this study}

- Randomised controlled trials ('randomised trials') can provide evidence on both the impact of interventions in general and differential impacts in different populations. This is critical for decisionmaking to address inequity.

- A consensus building process was used to develop and validate a conceptual framework to identify and classify health equity-relevant randomised trials.

- The proposed conceptual framework engages users in a deliberative process to design and report studies to provide evidence that is useful to understand the distribution of effects rather than only average effects.

- A limitation is that the analysis and publishing of disaggregated data can be expensive and may not be desirable for all randomised trials.

- The conceptual framework may be used to design health equity-relevant randomised trials (as well as other study types) and to identify health equityrelevant studies that contribute to an evidence base that improves overall health and health equity.

and between countries. ${ }^{1}$ For example, within Canada, the difference in life expectancy between Indigenous and general populations is on average 10 years. ${ }^{2}$ Differences in health between individuals or population groups within society, or health inequalities, are labelled 'inequities' when considered unfair. ${ }^{3}$ There are differing views on what counts as unfair and moral judgements about fairness may vary over time and between cultures. ${ }^{4}$ For example, men have a shorter life expectancy (with less disability) than women, and there is controversy about whether this is inequitable. ${ }^{5}$ Two common guiding principles, frequently advocated in public health 
policy documents, are that health inequalities can be considered unfair when they are potentially avoidable by organised social action and when the situation that causes poor health is out of the control of those affected. ${ }^{6}$ This includes situations where choices are severely restricted by social, historical, economical or built environments. The causes of health inequity are multidimensional and include factors described as social determinants of health and health sector factors. For the purposes of this paper, we choose to define health equity in this way to reflect our collective interests in social justice and the removal of social barriers posed by discrimination and stigmatisation which affect participation by individuals or populations in opportunities for better health.

Healthcare decisions in most countries are commonly guided by different types of evidence, among which clinical evidence based on randomised controlled trials ('randomised trials') usually holds particular prominence. To be useful for health equity policy, considerations for differences within and between groups and gradients of effects across socially stratifying factors are critical. Considering health equity is important to direct approaches that are either universal and intended to address health inequity for a whole population or that are focused and directed at a specific subgroup that experiences health inequity. Improved reporting of health equity in randomised trials is important as some interventions can aggravate rather than reduce inequities ('intervention-generated inequities'). ${ }^{7}$ A review of public health media campaigns for health behaviour revealed that health information provision was more effective in motivating behaviour change among socioeconomically advantaged groups and widened the observed gap in health behaviour between socioeconomically disadvantaged groups. ${ }^{7}$ Although improving average health is an important social goal, increased inequity is an undesirable side effect. The improved reporting of health equity implications of interventions is critical, and the increased use of randomised trial designs are promoted in the areas of public health ${ }^{8}$ and international development ${ }^{9}$ to build evidence that informs equity-oriented decisions.

Randomised trials provide evidence on the impact of an intervention and, in principle, can also assess their impact on health equity; however, randomised trials often fail to report relevant sociodemographic factors. ${ }^{10}$ In addition, subgroup analyses, even when appropriately powered are rarely conducted to assess whether the impact of the intervention differs across subgroups in society (eg, differential impact by socioeconomic status, place of residence). ${ }^{11}$ To address the poor reporting and analysis of equity impacts in randomised trials, we are assessing the need for additional reporting guidelines for randomised trials that provide evidence about potential impact on health equity. ${ }^{12}$ An extension of the internationally recognised Consolidated Standards for Reporting Trials (CONSORT) statement, an evidencebased guideline to encourage completeness and transparency of reporting in randomised trials ${ }^{13}$ is under way. The purpose of this paper is to describe the development and validation of a conceptual framework to identify when a randomised trial provides evidence about health equity. The conceptual framework may be helpful for the design and reporting of randomised trials by trialists, funders, journal editors and other knowledge user groups.

\section{METHODS}

We convened a collaborative research team and advisory board including interdisciplinary and international knowledge users in a deliberative, consensus building process as part of a larger study described elsewhere. ${ }^{12} \mathrm{We}$ searched for and did not find any frameworks to define an equity-relevant randomised trial. ${ }^{12}$ We then conducted a series of meetings that included representatives of potential knowledge user groups, including: bioethicists, clinicians, funders, journal editors, patient representatives, members of the public, policymakers, systematic review authors and trialists. We engaged in a process to define features of randomised trials that provide evidence about health equity, which we define as 'health equity-relevant' randomised trials.

As a first step, we agreed on an operational definition of health equity for our work on health equity in randomised trials, drawing on social determinants of health theory that describes how socially structured health disadvantage is experienced and perpetuated. ${ }^{14}$ We agreed, for this context, that the term 'health equity' implies that ideally everyone can attain their full health potential and that no one should be disadvantaged from doing so because of their social position or other socially determined circumstance. ${ }^{15}$ Health equity addresses both access to healthcare and the broader concept of opportunities to achieve good health. ${ }^{6}$ Moreover, we recognise that there are a range of characteristics of individuals or populations across which health inequities may exist and that reasonable people hold different views about what counts as unfair. ${ }^{16}$

Evidence shows that across the social determinants of health for individuals and population groups, people may experience disparities in health status, access to services and the quality of care received. ${ }^{14}$ Social determinants may be multifactorial and interact with one another. They can also be dependent on the context and setting, such as the local political or social climate and the form of health systems in which individuals and populations are situated. ${ }^{17}$ One organising framework is 'PROGRESS-Plus' that depicts social determinants of health and enables the examination of the relationships within and between the multiple, interacting and socially structured characteristics that may influence the opportunities for health of individuals and populations: Place of residence, $\mathbf{R a c e}^{\mathrm{i}}$ /ethnicity/culture/language, Occupation,

\footnotetext{
We adopt the American Anthropological Society (1998) statement on race: 'Present-day inequalities between so-called 'racial' groups are not consequences of their biological inheritance but products of
} 


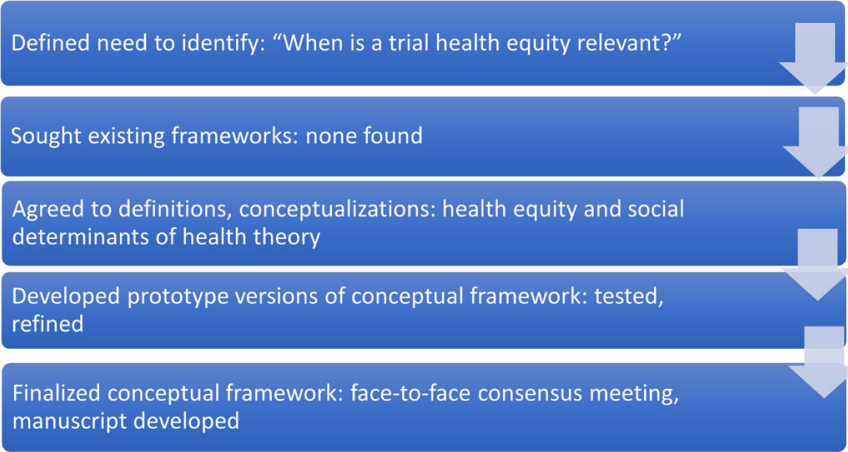

Figure 1 Description of the conceptual framework development process.

Gender/Sex, Religion, Education, Socioeconomic status, and Social capital. ${ }^{18-20}$ Due to advances in thinking about causal associations/linkages between degree of control in the living environment and socioeconomic inequities in health-related outcomes, 'Control' was included with 'Social Capital'. ${ }^{21}$ PROGRESS-'Plus' represents additional context-specific personal or setting characteristics that may be associated with health inequities and that are relevant to our study: (1) individual characteristics, for example, disability, age, sexual orientation; (2) features of relationships with effects on power differentials that impact personal autonomy, for example, children who suffer passive smoking because their parents smoke in the home and (3) time-dependent transitions, for example, migration and/or refugee status. ${ }^{22}{ }^{23}$ The degree to which PROGRESS-Plus characteristics are associated with health inequity depends on time, place and interaction between the different dimensions.

A conceptual framework was developed and feedback on the concepts and format of the conceptual framework was invited from over 40 clinicians and methodologists in face-to-face meetings, teleconferences and email. These communications used randomised trial examples to structure conversation and debate about the meaning of 'health equity relevant'. Furthermore, we gathered opinions from many others in local seminars and at international conferences. This feedback was incorporated through iterative adjustments to the conceptual framework.

The conceptual framework was tested with the research team and advisory board. ${ }^{12} \mathrm{~A}$ search for randomised trials was designed with a librarian scientist (JM) in MEDLINE, using text words and subject headings for the concepts of PROGRESS-Plus and a validated filter for randomised trials $^{24}$ (see online supplementary appendix A). We developed this search by testing whether it identified a reference set of 10 articles classified as health equity relevant according to our framework. ${ }^{12}$ The search was run from 2013 to 2015 and generated 4316 hits. Of these, 1025 were judged to be equity relevant based on title and abstract

historical and contemporary social, economic, educational, and political circumstances'. screening. These 1025 articles were arranged in random order, then screened in full text by two independent reviewers for eligibility using the criteria developed to define a health equity-relevant trial. We screened for eligibility until we identified 200 health equity-relevant trials, selected as the sample size for a concurrent methods study (in progress). The sample of trials was tested with the conceptual framework for consistency of concepts. Conversation and debate about concepts occurred and led to consensus around five aspects of randomised trials, and the definition was revised to reflect the consensus reached in these discussions. The research team and advisory board confirmed the final adjustments to the conceptual framework (figure 1).

\section{RESULTS}

A randomised trial is considered to provide health equity-relevant evidence if it actually or potentially assesses the effects of an intervention on the health or its determinants of either individuals or a population who experience ill health due to social disadvantage (figure 2). These randomised trials can either exclusively focus on individuals or populations experiencing socially structured disadvantage or consist of a mixed group and collect data potentially useful for assessing differential effects of the intervention across PROGRESS-Plus characteristics (table 1). The evidence from these randomised trials may be used to mitigate socially structured health disadvantage, that is, contribute to understanding of the extent to which the intervention in question is effective in population groups who experience disadvantage. To identify 200 health equity-relevant randomised trials, 643 articles were screened. Screening confirmed that the criteria for health equity relevance could be applied to randomised trials consistently.

Five aspects of randomised trials are reported as findings and reflect the debated points during the validation of the conceptual framework concepts. We considered five aspects of randomised trials and their relevance to define a randomised trial as health equity relevant: (1) type of interventions, (2) stated objective about equity, (3) estimation of differential impacts, (4) levels of health equity-relevant data and (5) setting and context.

\section{Type of intervention}

We agreed that the type of intervention (eg, pharmacological, non-pharmacological, health systems) was not relevant to identifying a randomised trial as health equity relevant. Although health equity is sometimes considered relevant only to public health or upstream interventions, we found many examples of clinical or pharmacological interventions that met our health equity-relevant definition. For example, we considered a randomised trial that evaluated a clinical intervention to promote asthma education for individual inner-city child patients with asthma that improved health outcomes ${ }^{25}$ to be health equity relevant. 
Does the study include individuals or a population that experience ill-health due to social disadvantage (defined across PROGRESS-Plus)?

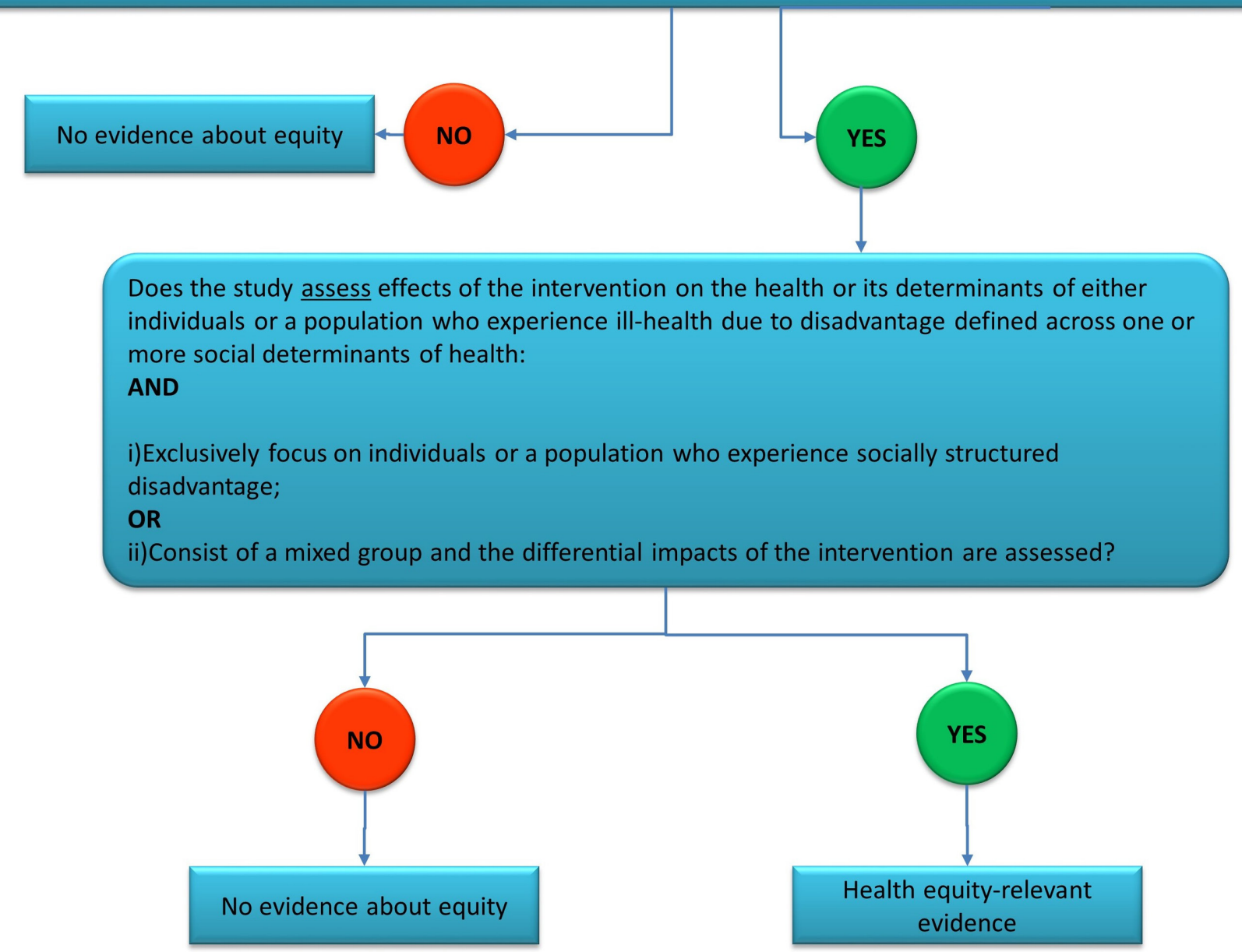

Figure 2 The conceptual framework.

Table 1 Examples of health equity-relevant studies

Includes individuals or a population that experiences ill health due to social disadvantage (defined across PROGRESS-Plus). Assesses the effects of the intervention on the health or its determinants of either individuals or a population who experience ill health due to disadvantage defined across one or more social determinants of health.

Either exclusively focus on individuals or a population or includes a mixed group

\section{'PROGRESS' criteria examples}

Assesses effectiveness of the intervention solely for groups who are considered to be at a social disadvantage

A trial of an intervention to prevent domestic violence in Indigenous communities. ${ }^{39}$
Assesses effectiveness of the intervention for groups considered to be at a social disadvantage compared with other groups considered to be at less of a disadvantage

A trial of an informed choice invitation on uptake of screening for diabetes in primary care assessed differences in effectiveness by socioeconomic status. $^{40}$

'Plus' criteria examples

Assesses effectiveness of the intervention solely for groups who are considered to be at a social disadvantage

A trial that tests a vocational A trial of a schooling intervention aimed at improving employment for people diagnosed with severe mental illness. ${ }^{41}$
Assesses effectiveness of the intervention for groups considered to be at a social disadvantage compared with other groups considered to be at less of a disadvantage conditional cash transfer programme on the behaviour of young women assessed effectiveness separately based on enrolment status at the start of the study: those who have dropped out versus stayed in school. ${ }^{42}$ 


\section{Stated objective about equity}

We found that a randomised trial did not need to have an explicit objective about improving health equity to be classified as health equity relevant. For example, a study that implemented a weight loss intervention for native Hawaiians and other Pacific Islanders did a secondary analysis of sociodemographic variables ${ }^{26}$ but did not identify health equity, inequalities or disparities as part of its objectives. However, the analysis of effects across income groups provides evidence about effectiveness of treatment for a population that is potentially disadvantaged in relation to opportunities for health.

\section{Estimate of differential impacts}

For the sake of transparency and to assess whether observed interactions are likely to result from multiple comparisons, it is critically important that an a priori plan of analysis is described that all examined interactions are reported on. Any post hoc subgroup analyses of randomised trial data (eg, across equity characteristics) are at risk of identifying spurious interactions and need to be interpreted with caution. There is, however, increasing recognition that data disaggregation is particularly important, such as analyses across sex/gender. ${ }^{27}$ The exploration of interactions should be situated within a theoretical perspective ${ }^{28}$ and/or empirical evidence as a base from which effect measure interactions may be assessed. For health equity, it is important to also consider gradients of effect across social stratifying factors such as income quintiles or with indices that reflect distribution such as the concentration index. ${ }^{29}$

Many randomised trials include a broad range of population groups, with limited details on sociodemographic characteristics and do not provide disaggregated data that could be used to assess whether effects of interventions differ between groups within the population. Furthermore, some randomised trials use detailed sociodemographic data to adjust analyses for baseline imbalances rather than deliberately exploring whether sociodemographic characteristics modify the effect of the interventions under study, thus losing the ability to assess similarities or differences in effects across these characteristics. ${ }^{30}$ This represents a missed opportunity for providing evidence about equity.

When trialists do not have a prespecified primary research objective of estimating differential impacts but nevertheless collect data on social determinants of health characteristics, then an opportunity exists to assess subgroup effects. The scope for a randomised trial to contribute health equity data may not be immediately apparent to the randomised trial designer. For example, even if each trial's statistical power to identify differential effects may be low, combining data from several trials (eg, in meta-analyses) may substantially enhance statistical precision to identify and estimate the strength of such interactions. Data from randomised trials can also be combined with data from observational studies or analysed with methods that incorporate explicit Bayesian priors and/or theories about differential impacts based on well-established bodies of evidence and theory. The problem of multiple tests and the effects on power can be addressed if all analyses of interaction are shown, as well as whether the interaction is defined as departure from 'multiplicativity' or 'additivity' or both. These interaction analyses should be described ${ }^{31}$ in the randomised trial registration and in the protocol paper. It is our view that opportunities to provide health equity-relevant evidence should be considered up front in randomised trial conception and design and reported transparently. Thus, collecting data about outcomes across social determinants of health may provide useful information for future and additional analyses, now greatly facilitated by global initiatives to allow data sharing. ${ }^{32}$

It is equally important to report analyses where no differences in effects are found since these may identify hypotheses for testing in future studies and any failure to identify effects due to low statistical power. If a health programme is equally effective in relative terms, if disadvantaged populations have a higher baseline risk, there will be a greater reduction in absolute events for disadvantaged populations. ${ }^{33}$ Such information is valuable for understanding the impact and potential for interventions to improve or exacerbate health inequities.

\section{Levels of health equity-relevant data}

According to our conceptual framework, an equity-relevant randomised trial may both focus on individuals and population experiencing socially structured disadvantage and collect data on differential impacts of the intervention in the form of subgroup effects, thus providing more than one level of health equity-relevant data. For example, a randomised trial of a video module that aimed to increase the use of a US poison centre by low-income parents is focused on a population experiencing social disadvantage, but it also provides evidence across language proficiency strata by conducting a subgroup analysis. ${ }^{34}$ The use of different intervention approaches is complementary and aims to address the multifactorial and intersecting characteristics of populations and individuals that affect health outcomes.

\section{Setting and context}

The population characteristics that influence socially structured disadvantage depend on the broader features of setting and context such as social, historical and political systems and require careful consideration. For example, a study with Japanese citizens conducted in Japan would not be classified as an equity-relevant randomised trial unless the participants were also considered to be disadvantaged across another dimension such as sex/gender or income.

We recognise that health equity concerns arise from an array of characteristics; judgments about particular individual or population health determinants are concerns for inequity and may be more difficult because of the need to carefully consider the context of the randomised trial 
and characteristics, such as age or disability. Studies that report interventions related to overcoming health disadvantage need to consider the relationship between the intervention and how it might relate to equity/inequity, such as an intervention to promote school enrolment and health outcomes between participants who have dropped out versus stayed in school. ${ }^{35}$

\section{DISCUSSION}

Operationalising a definition of a health equity-relevant randomised trial required consideration of broader issues and meanings of social justice informed by values, context and the impact of different social arrangements. Our conceptual framework engages users to consider and identify the many instances of health equity-relevant evidence and the potential contributions to broader societal dialogues. The evidence from these randomised trials may be used to mitigate socially structured health disadvantage, that is, contribute to understanding of the extent to which the intervention in question is effective in population groups who experience disadvantage.

The conceptual framework is proposed as a way to identify and operationalise understandings about how randomised trials can provide evidence about health equity and in particular complements two previous intervention frameworks related to improving health equity. Margaret Whitehead's typology is based on the underlying theory of change and guides consideration of appropriate application of interventions to address health inequity; interventions fall into one of four main categories: strengthening individuals, strengthening communities, improving living and working conditions and promoting healthy macropolicies. ${ }^{15}$ Hilary Graham's typology identifies three main approaches: addressing health inequity gaps, gradients or targeted approaches. ${ }^{36}$ Our conceptual framework builds on these classification systems and suggests ways randomised trials can provide health equity-relevant evidence for an intervention, even when providing evidence about equity is not the central aim. Our conceptual framework has potential applications to inform other study designs and is not limited to that of randomised trials.

It is our view that opportunities to provide health equity-relevant evidence should be considered up front in randomised trial conception and design and reported transparently. While different users will hold different definitions and priorities in regard to disadvantage and opportunities for health, we argue that consideration of individual or population characteristics and societal context is of paramount importance. Moreover, consideration of equity aligns with and supports an approach to decision-making that enables consideration of distributional goals and outcomes aimed at addressing health inequity ${ }^{36}$ Knowledge about differential effects (or lack thereof) using social determinants of health theory, operationalised across PROGRESS-Plus characteristics can contribute to build knowledge aimed at improved application of effective interventions.

The approach to health equity supported by the conceptual framework promotes thinking about randomised trials as more than just a means of developing evidence about average efficacy or effectiveness. It highlights opportunities for all randomised trials to contribute to a broader social agenda about reducing health inequity. In doing so, it upholds international policy on 'research for health' that advocates for the development of research methods to promote better knowledge and standardisation of reporting mechanisms. ${ }^{3738}$

\section{Limitations and strengths}

A limitation of this approach is that analysing and publishing disaggregated data can be expensive, and it is not desirable for all randomised trials to do subgroup analyses by PROGRESS-Plus characteristics. We propose that, when possible, data on equity-relevant characteristics be collected, even when the study may not have sufficient power to perform subgroup analyses. In this way, data about characteristics linked to health equity may be made available for other researchers to use in pooled analyses, modelling or systematic reviews. Additionally, other knowledge sources/systems are essential to understand health inequities and not all social determinants of health are a source of 'disadvantage'. As well, relationships within and between social determinants of health mean that there may be a need to explicitly identify the factors that are relevant and therefore have been focused on, as not every facet of inequity is relevant to every research question. The proposed conceptual framework, therefore, is designed to engage users in deliberation about designing and reporting studies that can provide evidence useful to understand distribution of effects, rather than only average effects. Strengths of the conceptual framework are the use of a collaborative approach to development and the iterative testing of the framework with examples of randomised trials.

\section{CONCLUSION}

The conceptual framework defines features of health equity-relevant trials that can be used to design and report both randomised trials and other study designs to improve the evidence base about how to improve health equity. Opportunities to provide health equity-relevant evidence should be considered upfront in trial concept and design. The conceptual framework can provide a stimulus to build knowledge about effects of interventions on health equity and is a first step to improve application of effective interventions and to build evidence that is of greatest relevance and use to individuals and populations.

\section{Author affiliations}

${ }^{1}$ Ottawa Hospital Research Institute, University of Ottawa, Ottawa, Ontario, Canada

${ }^{2}$ Department of Public Health and Policy, University of Liverpool, Liverpool, UK 
${ }^{3}$ Department of Social and Environmental Health Research, Faculty of Public Health and Policy, London School of Hygiene and Tropical Medicine, London, UK ${ }^{4}$ Centre for Research on Educational and Community Services, School of Psychology, University of Ottawa, Ottawa, Ontario, Canada

${ }^{5}$ Department of Social Science, Evidence for Policy and Practice Information and Co-ordinating Centre, Social Science Research Unit, University College London, London, UK

${ }^{6}$ Bruyère Continuing Care, Bruyère Research Institute, Elisabeth Bruyere Research Institute, University of Ottawa, Ottawa, Ontario, Canada

${ }^{7}$ The South African Cochrane Center, South African Medical Research Council, Cape Town, South Africa

${ }^{8}$ Faculty of Medicine and Health Sciences, Stellenbosch University, Stellenbosch, South Africa

${ }^{9}$ Rotman Institute of Philosophy, University of Western Ontario, Ontario, Canada

${ }^{10}$ Clinical Epidemiology Program, Ottawa Hospital Research Institute, Ottawa, Ontario, Canada

${ }^{11}$ School of Epidemiology, Public Health and Preventive Medicine, University of Ottawa, Ottawa, Ontario, Canada

${ }^{12}$ Research Ethics and Governance, University College London, London, UK

${ }^{13}$ Department of Clinical Epidemiology and Biostatistics, McMaster University, Hamilton, Ontario, Canada

${ }^{14}$ Biostatistics Unit, Father Sean O'Sullivan Research Centre, St Joseph's

Healthcare, Hamilton, Ontario, Canada

${ }^{15}$ Centre for Health Economics, University of York, York, UK

${ }^{16}$ Department of Medicine, University of Ottawa, Ottawa, Ontario, Canada

${ }^{17}$ Cochrane Musculoskeletal Group, Ontario, Canada

${ }^{18}$ Brandon University, Brandon, Manitoba, Canada

${ }^{19}$ Office of Knowledge Management, Bioethics and Research, Pan American Health Organization/World Health Organization, Washington, District of Columbia, USA

${ }^{20}$ Melbourne School of Population and Global Health, The University of Melbourne,

Melbourne, Victoria, Australia

${ }^{21}$ Campbell Collaboration, New Delhi, India

${ }^{22}$ Department of Family Medicine, Pontificia Universidad Catolica de Chile, Santiago,

Chile

${ }^{23}$ Centre for Intervention Science in Matnernal and Child Health (CISMAC), University of Bergen, Bergen, Norway

${ }^{24}$ Department of Global Public Health and Primary Health Care, University of Bergen, Bergen, Norway

${ }^{25}$ Department of Global Health, Milken Institute School of Public Health, George

Washington University, Washington, District of Columbia, USA

${ }^{26}$ Centre for International Health, University of Bergen, Bergen, Norway

${ }^{27}$ Department of Community Health and Epidemiology, Faculty of Medicine,

Dalhousie University, Halifax, Nova Scotia, Canada

${ }^{28}$ Department of Epidemiology and Community Medicine, University of Ottawa,

Ottawa, Ontario, Canada

${ }^{29}$ Ottawa Heart Institute, University of Ottawa, Ottawa, Ontario, Canada

${ }^{33}$ Norwegian Institute of Public Health, Oslo, Norway

Contributors $\mathrm{JJ}$ and VW conceived and led the design of the work described in the manuscript and were responsible for the first and final drafts of this manuscript. All authors participated in and provided substantial contributions to the analysis and interpretation during development of the work described in the manuscript. All authors made contributions to drafts of this manuscript and have reviewed and revised it for important intellectual content and approved the final version.

Competing interests None declared.

Provenance and peer review Not commissioned; externally peer reviewed.

Data sharing statement № additional data available.

Open Access This is an Open Access article distributed in accordance with the Creative Commons Attribution Non Commercial (CC BY-NC 4.0) license, which permits others to distribute, remix, adapt, build upon this work non-commercially, and license their derivative works on different terms, provided the original work is properly cited and the use is non-commercial. See: http://creativecommons.org/ licenses/by-nc/4.0/

(C) Article author(s) (or their employer(s) unless otherwise stated in the text of the article) 2017. All rights reserved. No commercial use is permitted unless otherwise expressly granted.

\section{REFERENCES}

1. World Health Organization. Rio political declaration on social determinants of health . Geneva $(\mathrm{CH})$ : World Health Organization, 2011. http://www.who.int/sdhconference/declaration/en/ (accessed 10 Dec 2016).

2. Statistics Canada. Life Expectancy. 2015 http://www.statcan.gc. $\mathrm{ca} / \mathrm{pub} / 89-645-\mathrm{x} / 2010001 /$ life-expectancy-esperance-vie-eng.htm (accessed 10 Dec 2016)

3. Whitehead M. The concepts and principles of equity and health. Int $J$ Health Serv 1992;22:429-45.

4. Eyal N. Inequalities in health: concepts, measures, and ethics: Oxford University Press, 2013.

5. Tsuchiya A, Williams A. A "fair innings" between the sexes: are men being treated inequitably? Soc Sci Med 2005;60:277-86.

6. Sen A. Why health equity? Health Econ 2002;11:659-66.

7. Lorenc T, Petticrew M, Welch V, et al. What types of interventions generate inequalities? Evidence from systematic reviews. $J$ Epidemiol Community Health 2013;67:190-3.

8. Macintyre S. Good intentions and received wisdom are not good enough: the need for controlled trials in public health. J Epidemiol Community Health 2011;65:564-7.

9. The Abdul Latif Jameel Poverty Action Lab (J-PAL). Introduction to evaluations. https://www.povertyactionlab.org/research-resources/ introduction-evaluations. (accessed 9 Aug 2016).

10. Furler J, Magin P, Pirotta M, et al. Participant demographics reported in "Table 1 " of randomised controlled trials: a case of "inverse evidence"? Int J Equity Health 2012;11:14.

11. Welch V, Petticrew M, Ueffing E, et al. Does consideration and assessment of effects on health equity affect the conclusions of systematic reviews? A methodology study. PLoS One 2012; 7:e31360.

12. Welch V, Jull J, Petkovic J, et al. Protocol for the development of a CONSORT-equity guideline to improve reporting of health equity in randomized trials. Implement Sci 2015;10:146.

13. Schulz KF, Altman DG, Consort MD. Statement: updated guidelines for reporting parallel group randomised trials. BMC Med 2010;2010:18.

14. Commission on Social Determinants of Health. CSDH final report: closing the gap in a generation: health equity through action on the social determinants of health. Geneva: World Health Organization, 2008.

15. Whitehead M, Dahlgren G, Mclntyre D. Putting equity center stage: challenging evidence-free reforms. Int J Health Serv 2007;37:353-61.

16. Daniels N, Kennedy BP, Kawachi I. Why justice is good for our health: the social determinants of health inequalities. Daedalus 1999;128:215-51.

17. Marmot MG. Policy making with health equity at its heart. JAMA 2012;307:2033-4.

18. Evans $\mathrm{T}$, Brown $\mathrm{H}$. Road traffic crashes: operationalizing equity in the context of health sector reform. Inj Control Saf Promot 2003;10:11-12.

19. O'Neill J, Tabish $\mathrm{H}$, Welch $\mathrm{V}$, et al. Applying an equity lens to interventions: using PROGRESS ensures consideration of socially stratifying factors to illuminate inequities in health. J Clin Epidemiol 2014;67:56-64.

20. Anon. AAPA statement on biological aspects of race. Am J Phys Anthropol 1996;101:569-70.

21. Whitehead M, Pennington A, Orton L, et al. How could differences in 'control over destiny' lead to socio-economic inequalities in health? A synthesis of theories and pathways in the living environment. Health Place 2016;39:51-61.

22. Oliver SKJ, Caird J , et al. Health promotion, inequalities and young people's health. A systematic review of research. London, UK: EPPI-Centre, Social Science Research Unit, Institute of Education, University of London, 2008.

23. Oliver S, Dickson K, Newman M. Getting started with a review. In: Gough SO D, Thomas J, eds. An introduction to systematic reviews London, UK SAGE Publications, 2012.

24. Haynes RB, McKibbon KA, Wilczynski NL, et al. Optimal search strategies for retrieving scientifically strong studies of treatment from Medline: analytical survey. BMJ 2005;330:1179.

25. Otsuki M, Eakin MN, Rand CS, et al. Adherence feedback to improve asthma outcomes among inner-city children: a randomized trial. Pediatrics 2009;124:1513-21.

26. Kaholokula JK, Townsend CK, Ige A, et al. Sociodemographic, behavioral, and biological variables related to weight loss in native Hawaiians and other Pacific Islanders. Obesity 2013;21:E196-E203.

27. Clayton JA, Collins FS. Policy: NIH to balance sex in cell and animal studies. Nature 2014;509:282-3.

28. Potvin L, Gendron S, Bilodeau A, et al. Integrating social theory into public health practice. Am J Public Health 2005;95:591-5. 
29. Razzaque A, Streatfield PK, Gwatkin DR. Does health intervention improve socioeconomic inequalities of neonatal, infant and child mortality? Evidence from Matlab, Bangladesh. Int J Equity Health 2007;6:4.

30. Morton RL, Schlackow I, Mihaylova B, et al. The impact of social disadvantage in moderate-to-severe chronic kidney disease: an equityfocused systematic review. Nephrol Dial Transplant 2016;31:46-56.

31. Sun X, Briel M, Busse JW, et al. Credibility of claims of subgroup effects in randomised controlled trials: systematic review. BMJ 2012;344:e1553.

32. AllTrials.2014. http://www.alltrials.net. (accessed 10 Dec 2016).

33. Anderson GM, Bronskill SE, Mustard CA, et al. Both clinical epidemiology and population health perspectives can define the role of health care in reducing health disparities. J Clin Epidemiol 2005:58:757-62.

34. Kelly NR, Harding JT, Fulton JE, et al. A randomized controlled trial of a video module to increase U.S. poison center use by low-income parents. Clin Toxicol 2014;52:54-62.

35. Baird SJ, Chirwa E, Jd H, et al. Girl power: cash transfers and adolescent welfare. Evidence from a cluster-randomized experiment in Malawi. NBER Work Pap Ser 2013;19479.
36. Graham $\mathrm{H}$. Tackling inequalities in health in England: remedying health disadvantages, narrowing health gaps or reducing health gradients? J Soc Policy 2004;33:115-31.

37. Pan American Health Organization. Policy on research for health: document CD49/10.49th Directing Council, 61st Session of the Regional Committee of WHO for the Americas. Washington DC: PAHO, 2009.

38. World Health Organization. The WHO strategy on research for health Geneva, Switzerland: WHO, 2012.

39. Andersson N, Shea B, Amaratunga C, et al. Rebuilding from resilience: research framework for a randomized controlled trial of community-led interventions to prevent domestic violence in aboriginal communities. Pimatisiwin 2010;8:61-88.

40. Marteau TM, Mann E, Prevost AT, et al. Impact of an informed choice invitation on uptake of screening for diabetes in primary care (DICISION): randomised trial. BMJ 2010;340:c2138.

41. Christensen TN, Nielsen IG, Stenager E, et al. Individual Placement and Support supplemented with cognitive remediation and work-related social skills training in Denmark: study protocol for a randomized controlled trial. Trials 2015;16:280

42. Baird S, Chirwa E, Mclntosh C, et al. The short-term impacts of a schooling conditional cash transfer program on the sexual behavior of young women. Health Econ 2010;19:55-68. 